\title{
Radiological/Nuclear Challenge Agent Source Type
}

National Cancer Institute

\section{Source}

National Cancer Institute. Radiological/Nuclear Challenge Agent Source Type. NCI

Thesaurus. Code C158318.

The mode by which the radiological or nuclear challenge agent is delivered to the subject. 